\title{
Milwaukee Shoulder Syndrome: An Extreme Case
}

\begin{abstract}
Nao Kuwahara, MD, Department of Internal Medicine; Kenichiro Tokunaga, MD, Department of Rheumatology; Michiko Nagamine, MD, Department of Pathology, Japanese Red Cross Kumamoto Hospital, Kumamoto City, Japan. Address correspondence to Dr. K. Tokunaga, 2-1-1, Nagamine-minami, Higashi-ku, Kumamoto-City 861-8520, Japan. Email: tokunaga.kenichiro.qx@kumamoto-med.jrc.or.jp. Informed consent from the proxy of the patient was obtained. We received approval of research ethics committee of Japanese Red Cross Kumamoto Hospital (approval number: No 19-41).
\end{abstract}

Milwaukee shoulder syndrome (MSS) usually occurs in elderly, especially female, patients and is a form of destructive arthropathy, often bilateral ${ }^{1}$. MSS is associated with the presence of basic calcium phosphate crystals (primarily hydroxyapatite) in the synovial fluid ${ }^{2}$.

An 82-year-old female presented with bilateral shoulder swelling and numbness of the left arm for 3 years. Medical history was unremarkable. Her shoulders were severely swollen, and her active range of motion was restricted to mild abduction. Radiographs showed the complete absence of the bilateral glenohumeral joints (Figure 1). Ultrasonography and magnetic reso-

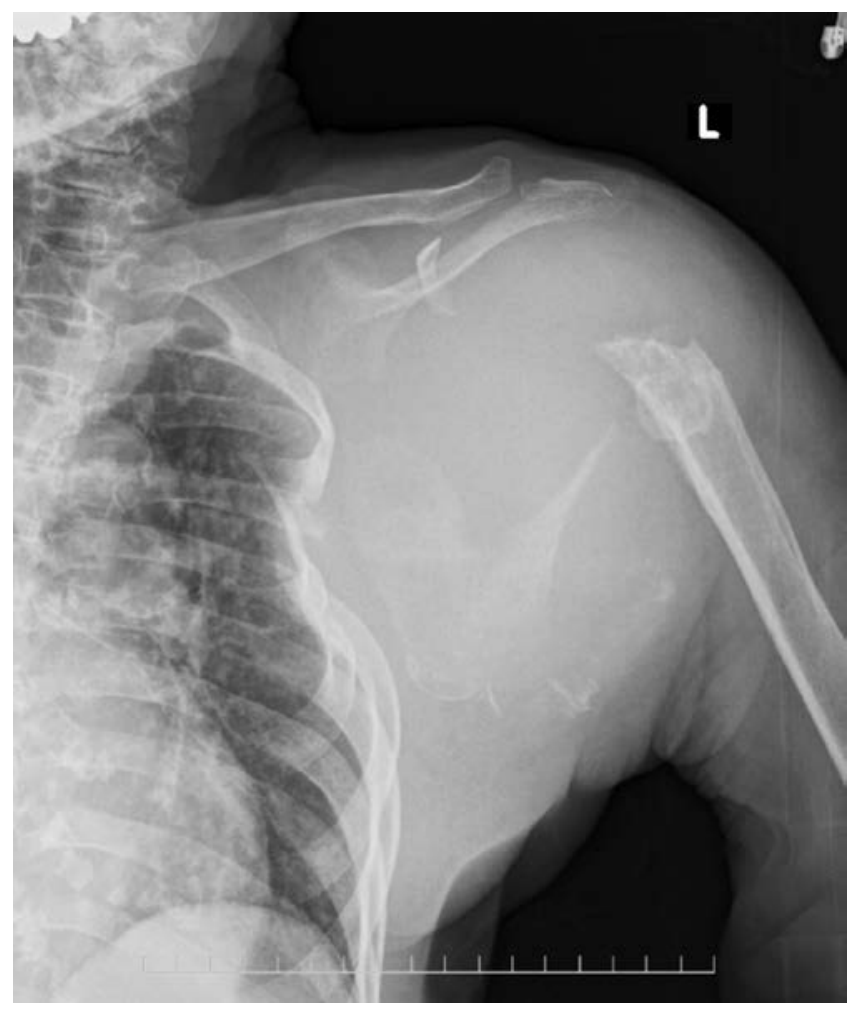

Figure 1. Radiograph shows the complete absence of the glenohumeral joint. nance imaging (MRI) of the shoulders revealed a mass suggestive of hematoma. MRI of the neck was unremarkable. Aspirated synovial fluid was hemorrhagic and the analysis was unremarkable (a few white blood cells and no organism by Gram stain; no crystals by polarized light microscopy). Alizarin red S staining revealed positive-stained materials consistent with hydroxyapatite crystals within the fluid. All cultures and PCR for Mycobacterium tuberculosis were negative, and several biopsies of the synovium revealed no malignancy. After extensive clinical workup to exclude differential diagnoses, the patient was diagnosed with MSS.

Treatment of MSS is supportive, with symptom relief as its goal. Shoulder joint replacement is an effective intervention but has to be performed before the rotator cuffs tear completely. There is a case report of a good response to colchicine and nonsteroidal antiinflammatory drugs ${ }^{3}$.

In this present case, the patient has been followed up with colchicine and rest, as there was no indication for arthroplasty. Six months later, she continued without any worsening pain.

\section{ACKNOWLEDGMENT}

We thank Editage (www.editage.jp) for English language editing.

\section{REFERENCES}

1. Stack J, McCarthy GM. Basic calcium phosphate crystal deposition disease. In: Hochberg MC, Gravallese EM, Silman AJ, Smolen JS, Weinblatt ME, Weisman MH, editors. Rheumatology 7 th ed. Philadelphia: Elsevier; 2018:1632-8.

2. McCarty DJ, Halverson PB, Carrera GF, Brewer BJ, Kozin F. "Milwaukee shoulder"--association of microspheroids containing hydroxyapatite crystals, active collagenase, and neutral protease with rotator cuff defects. I. Clinical aspects. Arthritis Rheum 1981;24:464-73.

3. Patel KJ, Weidensaul D, Palma C, Ryan LM, Walker SE. Milwaukee shoulder with massive bilateral cysts: effective therapy for hydrops of the shoulder. J Rheumatol 1997;24:2479-83. 